\title{
An Integrated Wearable Robot for Tremor Suppression with Context Aware Sensing
}

\author{
Denis Huen, Jindong Liu, and Benny Lo
}

\begin{abstract}
Tremor is a neurological disorder which can significantly impede the daily functions of patients. The available treatments for patients with tremor are mainly pharmacotherapy and neurosurgery, but these treatments often have side effects. A wearable exoskeleton can potentially provide the assistance needed for patients with Parkinsonian or essential tremor to carry out daily activities and enable independent living. This paper presents the design and development of a 3D printed lightweight tremor suppression wearable exoskeleton. One of the major technical challenges for wearable robot is to maintain long battery life meanwhile miniature in size for practical use. This paper proposes an integrated approach where context aware Body Sensor Networks (BSN) sensors are incorporated to characterize voluntary and tremor movement, and detect activities of daily life (ADL). With the contextual information, the system can determine the intention of the user, optimize its control and minimize its power consumption by providing the necessary suppression only when needed. The preliminary result has shown that the wearable robot prototype can reduce the amplitude of simulated tremor by around $77 \%$, and accurately identify different ADL with accuracy above $70 \%$.
\end{abstract}

\section{INTRODUCTION}

We can categorize human motions into voluntary and involuntary motion in general. Voluntary motion represents the intended movements, such as picking up a coffee mug, while involuntary motion is unintentional. An example of an involuntary motion is the tiny vibration of the arm and fist when you're trying to hold your fist tight and still. This is caused by the overcompensation of the feedback control mechanism of the musculoskeletal system. Although such involuntary motions are normal and have no effect on one's life, other involuntary movements, such as tremor, could have significant impacts on the wellbeing and health of a patient. Tremor is a rhythmic, involuntary muscular activation at an almost fixed frequency and amplitude, which is a symptom of several neurological disorders and neurodegenerative diseases, such as Parkinson's disease. Tremor commonly affects the upper limbs, but certain types of tremors can affect different parts of body, such as head, tongue and legs. There are mainly three types of tremor: resting, postural and action tremor[1]. Resting or static tremor occurs when the target muscle or muscle group is at

D. Y. F. Huen, J. Liu and B. Lo are with the The Hamlyn Centre for Robotic Surgery, Imperial College London, UK. (e-mails: \{y.huen14, j.liu, benny.lo\}@imperial.ac.uk).

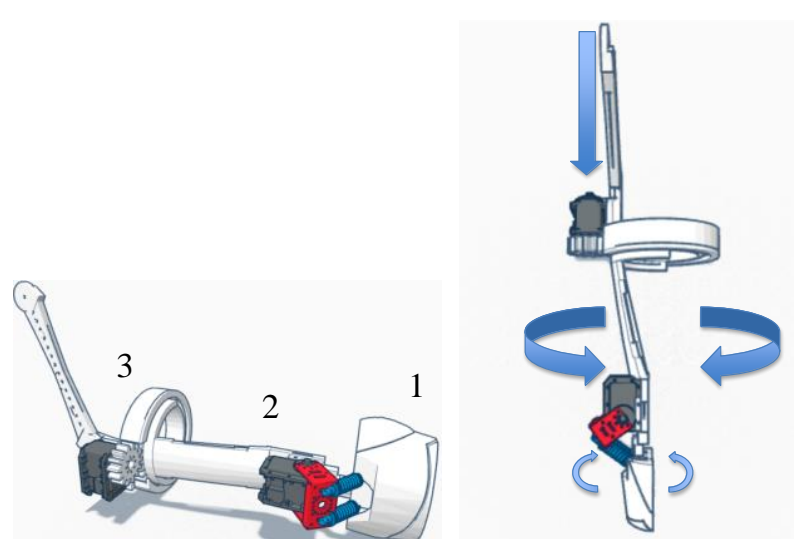

Figure 1 Concept design and main structure parts of the wearable robotic device with 2 DOF

rest, which is the commonly associated with Parkinson's disease. Postural tremor occurs when the muscle is holding in a position against gravity. In contrast, action tremor occurs when the target muscle or muscle group is activated. The tremor frequency is typically in the range of $3-12 \mathrm{~Hz}$, where frequency of resting tremor usually falls within the range of 3 $-7 \mathrm{~Hz}$ (up to $75 \%$ of patients with Parkinson's disease) and action tremor, on the hand, has a relatively higher frequency of $5-12 \mathrm{~Hz}$ (around $65 \%$ of patients with Parkinson's disease) [2-9]

Currently, the clinical treatments available for patients with tremor are mainly pharmacotherapy or neurosurgery through Deep Brain Stimulation (DBS). However, the effect of these treatments may deteriorate after prolonged use and could cause side effects, and not all the treatments are suitable for all patients. For instance, it was found that effect of medication on tremor suppression will wear off over time and may cause fatigue, sleepy, nausea, poor vision and muscle paralysis[10]. On the other hand, DBS has shown remarkable successes in removing tremors, and enabled many patients to return to normal healthy life. DBS reduces the tremor by stimulating targeted areas of the brain, such as globus pallidus internus and subthalamic nucleus, depends on the specific symptom [11, 12]. Despite the high risk of neurosurgery, DBS is only suitable for a small group of patients and may cause disturbances in behavior and cognition and cerebral hemorrhage [11, 12]. Less invasive approaches, such as exoskeleton and assistive devices, have been proposed as an alternative approach to attenuate tremor and assist daily living [13, 14]. Several wearable tremor suppression devices are proposed, for example, "Viscous Beam" [15], WOTAS [16-20], glove with 
piezoelectric materials [21, 22] or conducting polymer actuators [23] or magneto-rheological dampers [24] or electromagnetic friction brake [25] or functional electrical stimulation (FES) [26-29]. Many of these devices are bulky, heavy and too power demanding to become a practical wearable device for patients. Soft exoskeleton based on functional electrical stimulation (FES) may be a more promising approach to develop a practical lightweight wearable device for suppressing tremor. However, misalignments of the electrodes due to movements and muscle fatigue are major issues hindering the long term use of FES devices [30].

In this paper, a lightweight exoskeleton is proposed for tremor suppression. Similar to the WOTAS approach, electric motors are used as actuator in the exoskeleton to suppress tremoric motions, and the lightweight structure of the

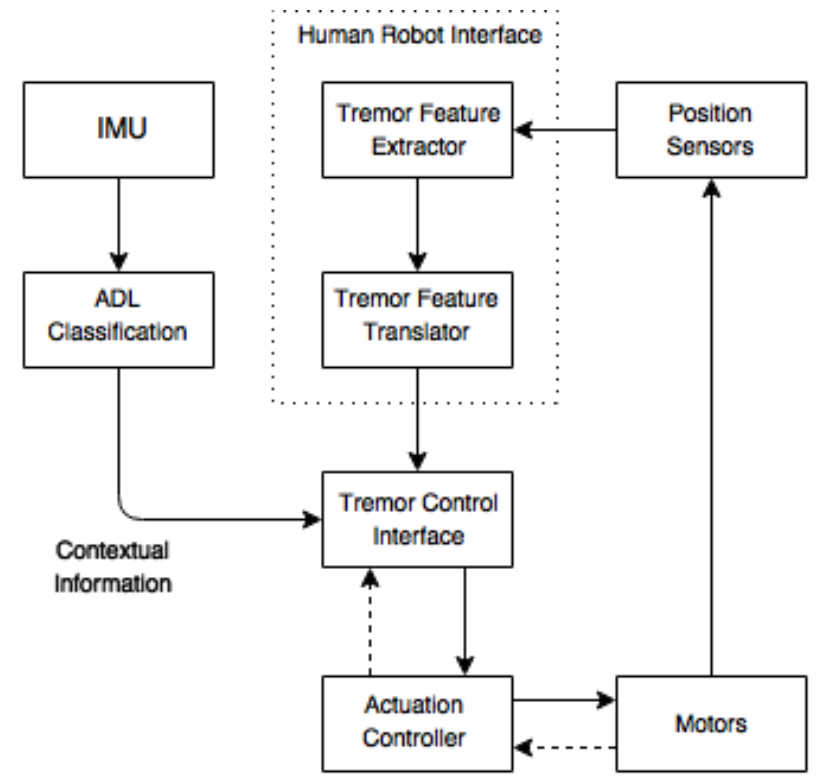

Figure 2 Control System of the proposed wearable robot

skeleton is built using rapid prototyping methods. To enhance user compliance and wearability of the robot, a new approach is proposed to incorporate the contextual information gathered from the detected intended activities into the robotic control to optimize the power consumption and provide the suppression only when needed.

\section{A ROBOTIC SYSTEM FOR TREMOR SUPPRESSION}

\section{A. System Specification}

With aim to alleviate the effects of tremor on daily living, a lightweight and low cost wearable exoskeleton robot was designed and a proof-of-concept prototype was developed. The mechanical structure of the robot was design and built using rapid prototyping technology, and the DYNAMIXEL and USB2Dynamixel (ROBOTIS, CA USA ) were selected as the actuator and motor control of the robot. The control strategy was programmed with $\mathrm{C} / \mathrm{C}++$ using Microsoft Visual Studio, and control commands are sent directly from the computer to the corresponding motors via the USB2Dynamixel (as shown in Figure 2).

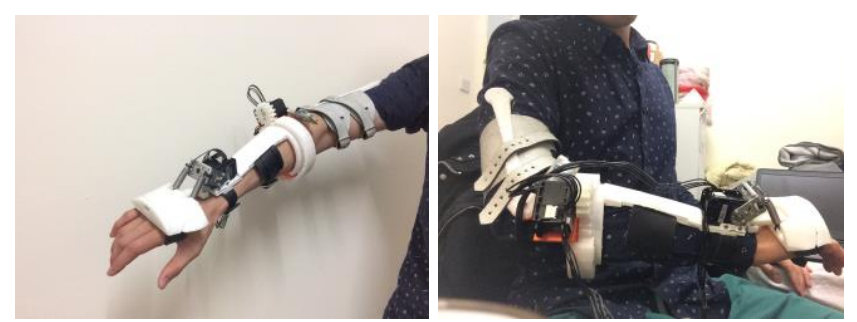

Figure 3 Final design and configuration of the wearable robotic device for the tremor control of the upper limb motions

\section{B. Control Strategy}

Prior research has shown that the pathological tremor frequency is usually with the range of $3 \mathrm{~Hz}$ to $12 \mathrm{~Hz}$ [2-9], while the frequency for daily activities is usually below 2 Hz. To effectively suppress tremor, the robotic control shall attenuate tremor motion with relatively higher frequency meanwhile bypass normal intended movements.

The control strategy we used in this study is a threshold approach.

Assume that the tremor motion is a sinusoidal wave pattern and the threshold frequency $h_{0}=3 \mathrm{~Hz}$ (with reference from previous researches.).

$$
\begin{aligned}
& \text { As } t=t_{0}, \quad p=p_{0} \\
& \text { As } \frac{d p}{d t}=0, \quad t=t_{1}
\end{aligned}
$$

Then Period $=4 \cdot\left(t_{1}-t_{0}\right)$

$$
\begin{gathered}
f=\frac{1}{\text { Period }}=\frac{1}{4 \cdot\left(t_{1}-t_{0}\right)} \\
\text { C }\left\{\begin{array}{c}
1 f \geq h_{0} \\
0 f \leq h_{0}
\end{array}\right.
\end{gathered}
$$

where $t$ is time, $p$ is position, $f$ is frequency, and $\mathrm{C}$ is the tremor control execution parameter.

The rate of change of position can be determined from the signals of position sensors inside the geared motors, and then the frequency of the motion is extracted. If the extracted frequency is above the threshold value of $3 \mathrm{~Hz}$, the suppression function will be activated accordingly. Actuation signal is sent to the geared motors via the controller to induce resistant force to dampen tremor motion. This control strategy is designed based on the assumption that tremor motion will have a higher frequency than voluntary motions. The embedded position sensors in the geared motors will continue sensing the positions/movements. The frequency will then be updated and input to the control system to adjust the counter movements to suppress tremor. The feedback control of the wearable robot is illustrated in figure 2 . 


\section{Wearable Device: Design and Mechatronics}

In order to validate the concept and design of the tremor suppression control strategy, a wearable robotic exoskeleton prototype was designed and developed as shown in figure 3. As a wearable robotic device, four key criteria of wearable device are considered in designing the robotic system: safety, robustness, ergonomics and reliability.

\section{Detection \& Actuation}

As a practical wearable robot has to react responsively in real time, detailed kinetic (interaction force between the limb and device) and kinematic (angular velocity) information has to be acquired and processed for active control. The DYNAMIXEL geared motors have built in sensors to provide real time measurement of position, speed, voltage, temperature, and status. Based on such readings from the motor, the system can obtain the accurate position, angular velocity and frequency of the motion of hand and forearm of the user while wearing the proposed robot.

\section{Material}

Instead of metallic structure, the structure of the proposed lightweight robot was designed and was $3 \mathrm{D}$ printed using a rapid prototyping machine with PLA and ABS plastic materials. The total weight of the prototype is about $350 \mathrm{~g}$. Unlike those exoskeletons designed for paraplegia which need to support the body weight of the users, exoskeleton for tremor suppression does not need the strength to support the body weight and the force required to suppress tremor is relatively low. 3D printing can enable construction of low cost personalized wearable robot.

\section{Structure}

The whole exoskeleton is formed by three parts: hand, forearm and upper arm with 2 degrees of freedom (DOF). The hand and the forearm sections of the exoskeleton are linked by a spring and metallic frame driven by the geared motor, while the forearm and the upper arm parts are linked with the mechanism of using spur gear driven by the geared motor. In figure 1, part 1 of the robot is designed for suppressing the tremor motion in flexion-extension of wrist while part 2 is designed for attenuating the tremor motion in pronation-supination of forearm. Part 3 of the structure is designed to be attached and fixed on the upper arm, and which will provide a fix point at the elbow joint. It will provide sufficient torque to stop the part 2 from turning and allow the suppression power to be transmitted through the forearm to the wrist more effectively.

\section{PERVASIVE SENSOR FOR DETECTING VOLUNTARY AND INVOLUNTARY MOVEMENTS}

A wearable robotic for tremor suppression should be lightweight and pervasive, and it needs to providing continue physical assistance throughout the day to support activities of daily living (ADL). Optimizing the power consumption of the system to provide long term assistance to patient is one of the major technical challenges in the design of practical wearable robot. Prior approaches mainly focus on optimizing the power consumption by using low power

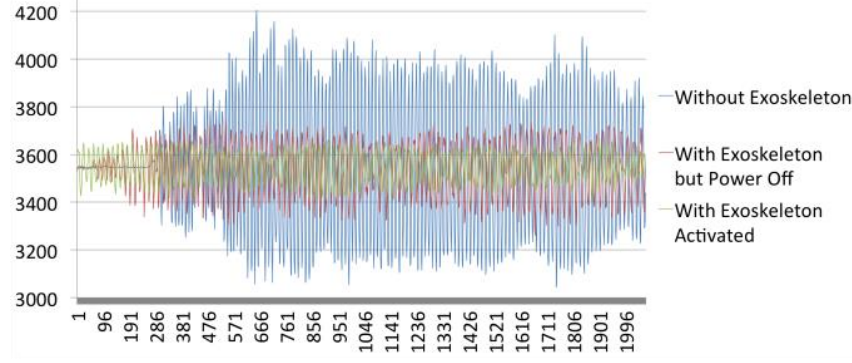

Figure 4 Raw signals from gyroscope detecting the simulated tremor motion acquired from the researcher.

electronics and actuators and optimize the robotic control. A new context aware approach is designed for the proposed wearable robot. By capturing and inferring the context, significant power reduction can be achieved by providing the necessary suppression only when needed. Tremor mainly affect certain delicate activities, such as drinking, eating, etc., it will not affect other normal activities, such as walking and sitting down. The tremor suppression is only needed for such fine activities. By activating the robot only for those activities, significant power saving can be achieved.

\section{A. Sensors}

In order to extract hand-wrist-forearm motion parameters for biomechanical analysis, a wireless Inertial Measurement Unit (IMU) sensor system based on the Body Sensor Networks (BSN) platform was used to capture the hand and forearm movements. BSN platform is a development platform designed specifically for BSN applications [31]. The system includes a wireless BSN node and a base station. A low power protocol was used for wirelessly transmission, where sensor data is sent in a 18-byte packet format with a frequency of $20 \mathrm{~Hz}$ [32].

With the aim of dampening tremor occurs at the wrist and hand, the BSN node with a tri-axial accelerometer and a triaxial gyroscope is designed to be positioned on the third metacarpal of the hand. The wrist flexion-extension and forearm pronation-supination movements were measured as they are the most important articulations in the kinematics of upper limb for tremor validation [20]. In order to attenuate the high frequency noise signal, the original signal collected from the sensors is first filtered with a second order low-pass Butterworth filter with cutoff frequency of $20 \mathrm{~Hz}$.

\section{B. Tasks}

To validate the proposed context aware design, an experimental of six different tasks was designed for detailed analysis. These six tasks are subsets of normal ADL chosen to test the capability of the system to distinguish different activities and demonstrate the functions of detecting the intended hand movements. Six healthy volunteers (three male and three female volunteers of the age from 20 to 28) were recruited and asked to perform all the tasks with and without the high frequency vibration motion (about $3 \mathrm{~Hz}$ ) (i.e. simulating tremor movements for 30 seconds each task in their own style), and the sensor signals from both 
gyroscopes and accelerometers were captured. Demonstration video of the tremor symptoms from a real patient was shown to each volunteer prior to the experiment. Since most of the tremor motions involve the flexionextension of wrist and the pronation-supination of forearm, according to prior clinical studies [33], the vibration motion at about $3 \mathrm{~Hz}$ of the flexion-extension of wrist is selected to simulate the tremor movement by the volunteer. The six tasks are 1) Sitting ,2)Walking, 3)Running, 4)Pointing an object, 5) Moving a bottle of water: grab a bottle of water and move it to the left and back to the original position, and 6) Holding a bottle of water while walking.
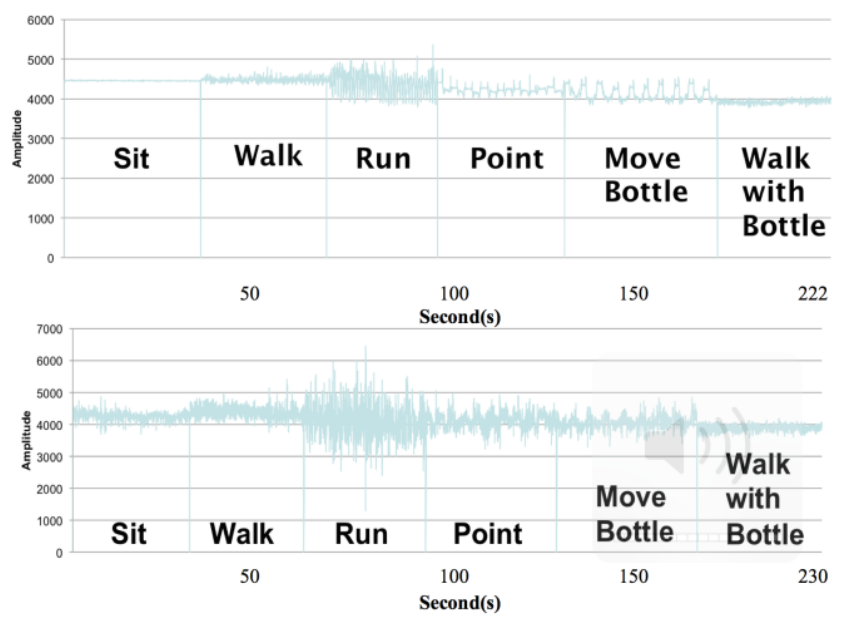

Figure 5 The signal magnitude vector (SMV) of the raw data from accelerometer for the six activities a) without and $b$ ) with simulated tremor motion.

Apart from Task 2, 3 and 6, the volunteer was sitting on a chair for all other tasks. All the tasks were recorded for 30 seconds. About 2050 data points were generated for each task in 30 seconds with the resolution of $0.014625 \mathrm{~s}$ and frequency at around $68 \mathrm{~Hz}$, and therefore there were 28,700 data points for each volunteer.

\section{Classification of Daily Activities}

\section{A. Feature extraction}

Data collected from the six volunteers were separated into six sets of data. In order to extract the relevant information from the continuous datasets for behavior recognition, the data sets were undergoing three basic processing steps: preprocessing, feature extraction and classification.

In the preprocessing step, a segmentation method for time series data "sliding windows" with $70 \%$ overlap was used to divide the signal into small time window of 1.6 seconds. With the statistical approach, seven different features for all windows were computed for the classification of the activities. The eight features are: i) Mean, ii) Standard deviation, iii) Skewness, iv) Kurtosis, v) Q1: the 25th percentile across the window (Q25), vi) Q2: the 50th percentile across the window (Q50), vii) Q3: the 75th
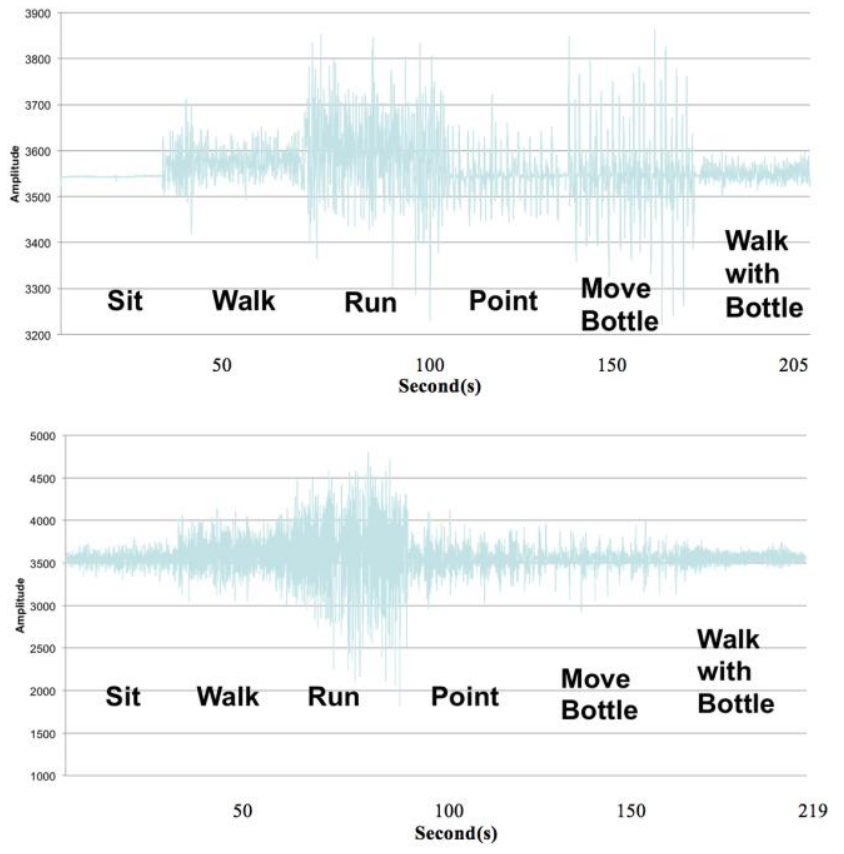

Figure 6 The signal magnitude vector (SMV) of the raw data from gyroscope for the six activities a) without and $b$ ) with simulated tremor motion.

percentile across the window (Q75), and viii) Inter-quartileRange (IR): $I R=Q 3-Q 1$

\section{B. Classification}

The selected features were used as the inputs to the activities recognition classifiers. In this study, to find a suitable activity recognition classifier for the wearable robot, a few most commonly used classifiers were evaluated, which include Naïve Bayes, Bayes Net, nearest neighbor, Random Forest, Sequential Minimal Optimization, Decision Tree J48, Decision table, RIPPER decision rules, Bagging and Adaboost M1 boosting were employed in WEKA environment, data mining open source software [34]. The performance of these behavior recognition classifiers were compared and evaluated. 10-fold cross-validation was employed for all the classifications to assess the performance of the classifiers.

\section{RESULT \& DISCUSSION}

\section{A. Wearable device: Deign and mechatronics}

To validate this novel concept and test this early wearable device prototype, the wearable robot was tested by the researcher. The BSN sensor was attached onto the position above the third metacarpal of the hand to record the motion signal from the gyroscope and accelerometer for evaluating the suppression function. Figure 4 illustrates the amplitude of the simulated tremor motion from the researcher before and after wearing the device without and with device being activated. The mean amplitude of simulated tremor motion was reduced by around $77 \%$ when comparing the signal of tremor before wearing the device and after wearing the device being activated. It is also observed that there was a 

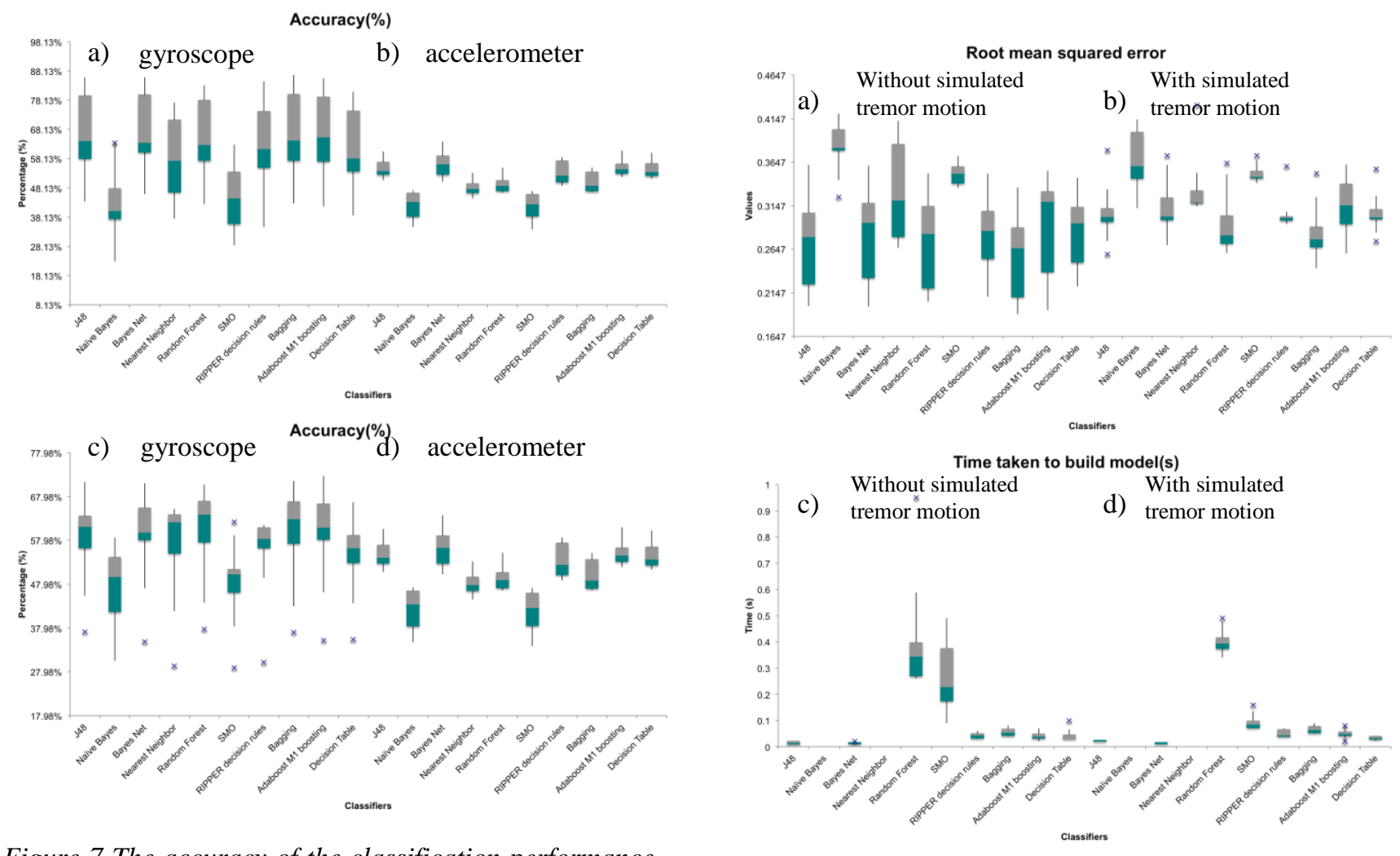

Figure 7 The accuracy of the classification performance of the activities by ten different classifiers for all six volunteers with the generated feature files from the signal of a) gyroscope and b) accelerometer, and those from the signal of c) gyroscope and d) accelerometer with simulated tremor motion.

significant reduction of tremor motion at about $65 \%$ in the amplitude when the device was worn but not activated. This was mainly caused by the geared motors inside the device and the frictions of the joints. Since there will be damping effect on the motion at the wrist and to the forearm by the geared motors due to the torque ratio that is equal to the gear ratio:

$$
R=\frac{T_{B}}{T_{A}}=\frac{N_{B}}{N_{A}}
$$

where $\mathrm{R}$ is the gear ratio, $\mathrm{T}_{\mathrm{A}}$ and $\mathrm{T}_{\mathrm{B}}$ are the input torque to the input gear and the output torque to the output gear correspondingly, and $\mathrm{N}_{\mathrm{A}}$ and $\mathrm{N}_{\mathrm{B}}$ are the number of teeth on the input gear and the number of teeth on the output gear. The torque generated from this gear ratio applies a damping force to the tremor motion without activating the tremor suppression system.

\section{B. Pervasive Sensing for distinguishing voluntary and involuntary movements}

Figure 5 and 6 shows the signal magnitude vector (SMV) of the raw data from accelerometer and gyroscope collected from one of the volunteers, which was calculated by the equation:

$$
A_{s m v}=\sqrt{A_{x}^{2}+A_{y}^{2}+A_{z}^{2}} \quad G_{s m v}=\sqrt{G_{x}^{2}+G_{y}^{2}+G_{z}^{2}}
$$

Figure 8 The classification performance in root mean squared error and time taken to build model of the activities by ten different classifiers for all six volunteers with the generated feature files from the signal of gyroscope a), c) without and b), d) with simulated tremor motion.

where $A_{s m v}$ is the signal magnitude vector (SMV) of the accelerometer, while $G_{s m v}$ is the signal magnitude vector (SMV) of the angular velocity. The reason of using the two SMVs rather than the raw sensor signals is that they are insensitive to orientation and position, and therefore various orientations and positions of the sensor will not need to be discriminated.

\section{Classification of daily activities}

Figure 7 shows the accuracy of the classification performance of the activities using ten different classifiers on inertial sensor data collected from all six volunteers. The average classification rate obtained by using gyroscope is better than those obtained using accelerometer. Thus, the features extracted from the gyroscope are sufficient for accurate activities classification. Figure 8 shows the root mean squared error and time taken to train the ten different classifiers based on the features extracted from the gyroscope. The graph shows results obtained from data collected when the volunteers were carrying out the activities with and without simulating tremor motion. By comparing the performance of all different classifiers being tested for the datasets without and with simulated tremor motion, Bayes Net, Random Forest, Decision Tree J48, Bagging and Adaboost M1 boosting show a relatively higher accuracy. 
Bagging produced the lowest root mean squared error during the classification, and Bayes Net classifier built the model in the shortest time. Among the different classifiers, Bagging classifier is the best classifier for the wearable robot, since it can produce a relatively high accuracy classification with lowest root mean squared error in a shorter time than other classifiers.

\section{CONCLUSION}

This paper presents a novel design and development of a 3D printed tremor suppression wearable exoskeleton. It proposed the integration of BSN sensors to detect the activities and behavior of the users with the aim of optimizing the control and minimizing the power consumption of the system. The mechanical structure and the working mechanism of the device with the threshold control strategy shows the success in suppressing the tremor motion simulated with a healthy subject. By introducing contextual sensing into the system, simulated tremor motion and voluntary motion can be captured and distinguished for optimizing the design of the tremor suppression control strategy. The preliminary result has shown that the wearable robot prototype can reduce the amplitude of simulated tremor by around $77 \%$, and accurately identify different activities with accuracy above $70 \%$.

\section{REFERENCES}

[1] S. M. 25. (2016). Involuntary Movements. Available: http://stanfordmedicine25.stanford.edu/the25/im.html

[2] R. N. Stiles and J. Randall, "Mechanical factors in human tremor frequency," Journal of Applied Physiology, vol. 23, pp. 324-330, 1967.

[3] R. J. Elble and J. E. Randall, "Mechanistic components of normal hand tremor," Electroencephalography and clinical neurophysiology, vol. 44, pp. 72-82, 1978 .

[4] A. Anouti and W. C. Koller, "Tremor disorders. Diagnosis and management," Western journal of medicine, vol. 162, p. 510, 1995.

[5] W. T. Ang, C. N. Riviere, and P. K. Khosla, "Design and implementation of active error canceling in hand-held microsurgical instrument," in Intelligent Robots and Systems, 2001. Proceedings. 2001 IEEE/RSJ, pp. 1106-1111.

[6] B. Pellegrini, L. Faes, G. Nollo, and F. Schena, "Quantifying the contribution of arm postural tremor to the outcome of goal-directed pointing task by displacement measures," Journal of neuroscience methods, vol. 139, pp. 185-193, 2004

[7] J. Zhang, F. Chu, and N. Mohammed, "DSP controller based signal processing of physiological hand tremor," in American Control Conference, 2005. Proceedings of the 2005, 2005, pp. 1569-1574.

[8] A. Izworski, M. Michałek, R. Tadeusiewicz, M. Rudzińska, J. Bulka, and I. Wochlik, "Acquisition and interpretation of upper limbs tremor signal in Parkinsonian disease," in Proceedings of the 4th WSEAS International Conference on ELECTRONICS, SIGNAL PROCESSING and CONTROL, Rio de Janeiro, Brazil, 2005.

[9] M. Engin, "A recording and analysis system for human tremor," Measurement, vol. 40, pp. 288-293, 2007.

[10] J. V. Campellone. (2014). Parkinson disease. Available: http://pennstatehershey.adam.com/content.aspx?productId=10\&pid=1 $0 \&$ gid $=000051$

[11] Y. Katayama, T. Kano, K. Kobayashi, H. Oshima, C. Fukaya, and T. Yamamoto, "Difference in surgical strategies between thalamotomy and thalamic deep brain stimulation for tremor control," Journal of neurology, vol. 252, pp. iv17-iv22, 2005.

[12] E. D. Flora, C. L. Perera, A. L. Cameron, and G. J. Maddern, "Deep brain stimulation for essential tremor: a systematic review," Movement Disorders, vol. 25, pp. 1550-1559, 2010.
[13] G. Deuschl, P. Bain, and M. Brin, "Consensus statement of the Movement Disorder Society on tremor," Movement Disorders, vol. 13, pp. 2-23, 1998.

[14] D. Sirisena and D. R. Williams, "My hands shake: Classification and treatment of tremor," Australian family physician, vol. 38, p. 678, 2009.

[15] J. Kotovsky and M. J. Rosen, "A wearable tremor-suppression orthosis," Journal of Rehabilitation Research and Development, vol. 35, pp. 373-387, 1998.

[16] M. Manto, M. Topping, M. Soede, J. Sanchez-Lacuesta, W. Harwin, J. Pons, J. Williams, S. Skaarup, and L. Normie, "Dynamically responsive intervention for tremor suppression," Engineering in Medicine and Biology Magazine, IEEE, vol. 22, pp. 120-132, 2003.

[17] E. Rocon, A. Ruiz, J. Pons, J. M. Belda-Lois, and J. SánchezLacuesta, "Rehabilitation robotics: a wearable exo-skeleton for tremor assessment and suppression," in Robotics and Automation, 2005. Proceedings of the 2005 IEEE ICRA2005, pp. 2271-2276.

[18] M. Manto, E. Rocon, J. Pons, J. M. Belda, and S. Camut, "Evaluation of a wearable orthosis and an associated algorithm for tremor suppression," Physiological measurement, vol. 28, p. 415, 2007.

[19] E. Rocon, M. Manto, J. Pons, S. Camut, and J. M. Belda, "Mechanical suppression of essential tremor," The Cerebellum, vol. 6, pp. 73-78, 2007.

[20] E. Rocon, J. Belda-Lois, A. Ruiz, M. Manto, J. C. Moreno, and J. Pons, "Design and validation of a rehabilitation robotic exoskeleton for tremor assessment and suppression," Neural Systems and Rehabilitation Engineering, IEEE Transactions on, vol. 15, pp. 367378, 2007.

[21] L. Swallow and E. Siores, "Tremor Suppression Using Smart Textile Fibre Systems," Journal of Fiber Bioengineering and Informatics, vol. 1, pp. 261-266, 2009.

[22] S. Kazi, A. As' Arry, M. M. Zain, M. Mailah, and M. Hussein, "Experimental implementation of smart glove incorporating piezoelectric actuator for hand tremor control," WSEAS Transactions on Systems and Control, vol. 5, pp. 443-453, 2010.

[23] S. Skaarup, L. Bay, and K. West, "Polypyrrole actuators working at 2 30Hz," Synthetic metals, vol. 157, pp. 323-326, 2007.

[24] D. Case, B. Taheri, and E. Richer, "Design and characterization of a small-scale magnetorheological damper for tremor suppression," Mechatronics, IEEE/ASME Transactions on, vol. 18, pp. 96-103, 2013.

[25] G. Herrnstadt and C. Menon, "On-Off Tremor Suppression Orthosis with Electromagnetic Brake," Journal ISSN, vol. 1929, p. 2724, 2013.

[26] D. Zhang and W. T. Ang, "Reciprocal EMG controlled FES for pathological tremor suppression of forearm," in Engineering in Medicine and Biology Society, 2007. EMBS 2007. 29th Annual International Conference of the IEEE, 2007, pp. 4810-4813.

[27] F. Widjaja, C. Y. Shee, D. Zhang, W. T. Ang, P. Poignet, A. Bo, and D. Guiraud, "Current progress on pathological tremor modeling and active compensation using functional electrical stimulation," in ISG'08: The 6th Conference of the International Society for Gerontechnology, 2008, pp. 001-006.

[28] L. P. Maneski, N. Jorgovanović, V. Ilić, S. Došen, T. Keller, M. B. Popović, and D. B. Popović, "Electrical stimulation for the suppression of pathological tremor," Medical \& biological engineering \& computing, vol. 49, pp. 1187-1193, 2011.

[29] J. A. Gallego, E. Rocon, J. Ibanez, J. L. Dideriksen, A. Koutsou, R. Paradiso, M. B. Popovic, J. B. Lois, F. Gianfelici, and D. Farina, "A soft wearable robot for tremor assessment and suppression," in Robotics and Automation (ICRA), 2011 IEEE, pp. 2249-2254.

[30] D. Tepavac and L. Schwirtlich, "Detection and prediction of FESinduced fatigue," Journal of Electromyography and Kinesiology, vol. 7, pp. 39-50, 1997.

[31] J. Ellul, B. Lo, and G. Yang, "The BSNOS platform: a body sensor networks targeted operating system and toolset," in The fifth international conference on sensor technologies and applications (SENSORCOMM 2011), 2011, pp. 381-6.

[32] B. P. Lo. (2006). BSN node Specifications. Available: http://ubimon.doc.ic.ac.uk/bsn/index.php?article=926

[33] K. W. Elble RJ, Tremor: Baltimore: Johns Hopkins University Press, 1990.

[34] M. Hall, E. Frank, G. Holmes, B. Pfahringer, P. Reutemann, and I. H. Witten, "The WEKA data mining software: an update," $A C M$ SIGKDD explorations newsletter, vol. 11, pp. 10-18, 2009. 